Artigo

\title{
Estudo de grupos sangüíneos em doadores de sangue caucasóides e negróides na cidade de São Paulo
}

Marcia C. Z. Novaretti ${ }^{1}$

Pedro E. Dorlhiac-Llacer ${ }^{2}$

Dalton A.F. Chamone

\begin{abstract}
Apesar do fato de que o grupo de mulatos representa um dos mais comuns grupos raciais en contrados não só no Brasil, mas em vários outros países, existe pouca informação sobrea distribuição dos gru pos sangüín eos nestes indivíduos. Foram estudados 2.462 doadores de sangue classifica dos como caucasóides, mulatos e negros conforme suas características antropológicas, bem como pela suasinformações sobre seus ancestrais. Foram estudadas as freqüências fenotípicas para os sistemas de grupos sangüín eos ABO, Rh, P, Kell, Rh, Lutheran, Lewis, Duffy e Kidd. Não foram encontradas diferenças estatisticamente significativas en tre negros e mulatos para a maioria dos sistemas de grupos sangüín eos por nós estudados, com exceção dos seguintes fenótipos: $P_{1}$ positivo, Dccee, Le( $\left.a-b-\right)$, Js( $\left.a+b+\right)$, Js(a$b+)$, Fy(a-b-), Fy(a+b+) e Fy(a-b+). Por outro lado foi observada uma diferença estatisticamente significante entre caucasóides e negróides para os seguintes fenótipos eritrocitários: $A, B, M+N-S+S$, $\mathrm{M}+\mathrm{N}-\mathrm{S}-\mathrm{s}+, \mathrm{P}_{1}$ positivo, ddccee, Dccee, Dccee, DCCee, DccEe, $\mathrm{K}+\mathrm{k}+$, $K-k+, K p(a-b+), K p(a+b+), J s(a-b+), J s(a+b-)$, Le$(a-b+)$, Le(a-b-), Fy(a$b+), F y(a+b+), F y(a-b-), J k(a+b-), J k(a+b+)$ eJk( $a-b+)$. Os resultados encontrados para o grupo mulato foram intermediários entre caucasóides e negros, com forte influência negróide.

Rev.bras.hematol.hemoter., 2000, 22(1):23-32
\end{abstract}

Palavras-Chave: grupos sangüíneos, estatística, doadores de sangue/dados numéricos

\section{Introdução}

A partir de 1500, com a descoberta do Brasil tivemos expressiva migração de portugueses, espanhóis, negros e mais recentemente, no início do século $X X$, de italianos, alemães e japoneses (1). Além da migração externa, existiu e existe significativa migração interna. Estas migrações ao longo do tempo contribuíram para um alto percentual de miscigenação (2) fazendo da população brasileira única do ponto de vista antropológico. Apesar do fato de que os Mulatos (resultantes da mistura entre Caucasóides e Negros) representam uma das misturas raciais mais comumente encontradas não só no Brasil (3),

1 - Doutora em Hematologia e Hemoterapia pela Faculdade de Medicina da Universidade de São Paulo, Chefe da Divisão de Imunohematologia e Agências Transfusionais da Fundação Pró-Sangue/Hemocentro de São Paulo 2 - Professor Livre Docente em Hematologia e Hemoterapia pela Faculdade de Medicina da Universidade de São Paulo. Diretor Técnico-Científico da FPS/HSP

3 - Professor Titular em Hematologia e Hemoterapia da Faculdade de Medicina da Universidade de São Paulo. Presidente da FPS/HSP

Endereço para correspondência: Av. Dr. Eneas de Carvalho Aguiar, 155. $1^{\circ}$ andar.

São Paulo. SP. CEP: 05403-000. Fone (11) 3061-5544. e-mail: marcia_novaretti@uol.com.br 
mas em muitos outros países, existe pouca informação a respeito da distribuição de grupos sangüíneos nestes indivíduos.

No B rasil existem inúmeros dados relativos ao estudo dos fenótipos eritrocitários na população indígena, em especial quanto aos sistemas $A B O$ e $\operatorname{Rh}(4,5,6,7)$. Entretanto, Salzano em 1971 (1), já referia que as informações relativas ao polimorfismo eritrocitário na população brasileira eram insatisfatórias tanto em número como em qualidade, e indicava a necessidade da realização de pesquisas outras inclusive para 0 sistema $A B O$ e Rh. Desde então, vários trabalhos têm sido publicados a nível nacional quanto aos sistemas de grupos sangüíneos $A B O$, Rh e MNS $(8,9)$. Para outros sistemas de grupos sangüíneos tais como Kell, Kidd, Duffy, Lewis, Lutheran e P, as informações são escassas (2, 10) sendo poucos os trabalhos com número significativo de indivíduos estudados com metodologia clara e precisa $(11,12)$.

O conhecimento da freqüência fenotípica dos vários grupos sangüíneos na nossa população é essencial para estimar a disponibilidade de sangue compatível para pacientes que apresentem anticorpos antieritrocitários. Permite ainda conhecimento sobre a influência indígena, oriental e negra na expressão dos antígenos eritrocitários.

0 objetivo deste estudo é o de conhecer em doadores de sangue caucasóides e negróides da Fundação Pró-Sangue / Hemocentro de São Paulo a freqüência fenotípica dos grupos sangüíneos ABO, Lewis, P, Rh, Kell, Kidd, MNS e Lutheran, assim como identificar se existem diferenças significativas entre os grupos estudados quanto aos fenótipos eritrocitários.

\section{Casuística e Métodos}

\section{Seleção dos Doadores}

Foram estudados 2.462 doadores de sangue, voluntários, de ambos os sexos, da Fundação Pró-Sangue/Hemocentro de São Paulo, entre 1991 e 1994. Os participantes foram incluídos nesta pesquisa após explanação dos objetivos da pesquisa e de consentimento individual por escrito.
Foram obtidas informações sociais de cada participante da pesquisa (nome, data de nascimento, idade, sexo), procedência, procedência dos pais e avós, número de doador, endereço, bairro, CEP, telefone (residencial e comercial), profissão, antecedentes transfusionais, uso de medicamentos e antecedentes gestacionais (para o sexo feminino).

Participaram desta pesquisa os candidatos à doação considerados aptos após triagem clínica e laboratorial, de acordo com os critérios para aceitação de doadores de sangue da Fundação Pró-Sangue/Hemocentro de São Paulo e Normas Técnicas do Ministério da Saúde para Coleta, Processamento e Transfusão de Sangue, Componentes e Derivados em Hemoterapia (13).

Os participantes deste estudo foram classificados em caucasóides e negróides de acordo com as suas características antropológicas (pigmentação da pele, tipo de cabelo, lábio e nariz) $(14,15)$ e pela informação a respeito dos ancestrais (pai, mãe, avós paternos e maternos) quanto à procedência e às características antropológicas dos mesmos (16). 0 grupo negróide foi subdividido em mulatos e negros. Foi classificado como caucasóide aquele que apresentava todas as características antropológicas observadas e to dos os ancestrais estudados caucasóides. Foi classificado como negro aquele que apresentava todas as características negróides e todos os ancestrais negros. Foi classificado como mulato aquele que apresentava pelo menos uma característica antropológica negróide, mas não todas, e/ou pelo menos um de seus ancestrais estudados era negro, mas não todos. Os doadores de sangue de raça amarela não foram estudados pois correspondem a menos que $0,5 \%$ do total dos doadores de sangue da Fundação PróSangue/Hemocentro de São Paulo (Cliquet ${ }^{17}$, 1990). Todos os participantes desta casuística foram entrevistados pelo mesmo observador.

Foram excluídos deste estudo: todos aqueles indivíduos que não conseguiram fornecer informações precisas referentes aos seus ancestrais; ou quando tivesse história de que algum parente havia doado sangue na FPS/ HSP após o início da pesquisa; e todo e qualquer 
doador de sangue com resultado alterado para os testes de triagem sorológica.

Todos os dados coletados assim como os resultados laborato riais foram armazenados em um software especialmente desenvolvido para este fim. Este programa possibilita a recuperação de qualquer informação inserida, quer isolada ou associada a outros dados.

\section{Testes Laboratoriais}

Todos os testes laboratoriais imunohematológicos foram realizados no Depto. de Imunohematologia da Fundação Pró-Sangue/ Hemocentro de São Paulo. A tipagem eritrocitária foi realizada emprego da técnica em tubo (18), usando reagentes anti- $A$, anti- $B$ e anti- $A B$ e reagentes de hemácias $A 1$ e $B$ (Biotest $S / A, B$ rasil). Anti-soros contra os antígenos $D, C, C, E, e, C^{\text {w }}$ $M, N, S, s K, k, K p^{a}, K p^{b}, J s^{a}, J s^{b}, J k^{a}, J k^{b}, L e^{a}, L e^{b^{\prime}}$, $P, L u^{a}$ e Lu ${ }^{b}$ (Biotest AG, Alemanha), foram empregados conforme recomendações do fabricante. A tipagem dos antígenos A, B, Le', $L^{b}, P, M$ e $N$ foi feita com leitura à temperatura ambiènte. Para a tipagem Rh1, nós empregados dois tipos de reagentes anti-D de diferentes procedências (soro anti-D policlonal, Biotest $S$ / $A$, Brasil e soro anti-D "blend", Biotest $A / G$, Alemanha), com leitura à temperatura ambiente e em fase de antiglobulina indireta. Todos os outros antígenos foram testados em fase de antiglobulina indireta após 30 minutos de incubação. Todos os testes laboratoriais foram realizados dentro de 24 horas após a coleta. Enquanto eram aguardados os resultados dos testes sorológicos para doenças transmissíveis pelo sangue, as amostras foram mantidas a $4^{\circ} \mathrm{C}$.

\section{Análise Estatística}

As freqüências dos diversos fenótipos nos grupos raciais estudados foram comparadas através do teste do qui-quadrado de Pearson (19) em tabelas de contingência. 0 valor crítico do nível de significância por nós preestabelecido foi de 0,05. Sempre que em um teste de comparação entre mais de dois grupos fornecia um resultado correspondentes a pelo menos essa probabilidade, dava-se continuidade à análise, através da partição da tabela de contingência inicial, com a finalidade de se tentar localizar quais dos grupos diferiam entre si.

\section{Resultados}

As freqüências fenotípicas são apresentadas nas tabelas 1-3 (freqüências relativas). A tabela 1 lista o percentual de indivíduos estudados para os sistemas ABO, MNS, P, Lutheran, Kell, Lewis, Duffy e Kidd em doadores de sangue caucasóides, mulatos e negros. As tabelas 2 e 3 mostram as freqüências fenotípicas dos sistemas de grupos sangüíneos MNS e Rh respectivamente.

\section{Discussão}

Apesar de estarem disponíveis atualmente técnicas moleculares para o estudo de grupos sangüíneos em alguns serviços de hemoterapia, até 0 momento não estas não são utilizadas rotineiramente, tendo sido aplicadas em casos especiais de investigação ou no estudo de pequenas populações, devido seu alto custo e complexidade técnica. Portanto, o conhecimento das freqüências fenotípicas dos principais sistemas de grupos sangüíneos em doadores de sangue brasileiros, caucasóides e negróides com atenção para o subgrupo mulato, pode contribuir para o cálculo da probabilidade de encontro de unidades negativas para determinado fenótipo.

Existem inúmeros trabalhos sobre os grupos sangüíneos em indígenas no $(6,7,20,21,22$, 23). Em indivíduos brancos e negros vários estudos referentes aos sistemas de grupo sangüíneo $A B O$ e $\mathrm{Rh}$ foram realizados em diversas cidades brasileiras, como São Paulo, Rio de Janeiro, Porto Alegre, Belém e Aracaju, dentre outras (10), porém, são escassos os dados em mulatos $(2,24)$ ainda que se saiba que os mulatos representam cerca de $55 \%$ da população brasileira.

Q uanto aos sistemas de grupos sangüíneos Lewis, P, Kell, Kidd, Duffy e Lutheran existem poucos trabalhos em nível nacional, a maioria realizada com pequeno número participantes, muitas vezes sem clara descrição da metodologia empregada, e/ou sem especificar como a classificação racial foi feita, prejudicando a análise dos resultados observados (10).

No nosso estudo a freqüência fenotípica para o sistema de grupo sangüíneo $A B O$, em caucasoídes e negros estava dentro dos valores já publicados anteriormente, no Brasil e em população européia $(10,24)$. 
Tabela 1. Freqüên cias fen otípicas dos sistemas de grupos sangüín eos ABO, P, Lewis, Kell, Kidd, Duffy, e Lutheran em doadores de sangue Caucasóides, Mulatos e Negros

\begin{tabular}{|c|c|c|c|c|c|c|c|c|c|}
\hline & \multicolumn{6}{|c|}{ População estudada } & \multicolumn{3}{|c|}{ TOTAL } \\
\hline \multirow[t]{2}{*}{$\begin{array}{l}\text { Sistema de Grupo } \\
\text { Sangüíneo }\end{array}$} & \multicolumn{2}{|c|}{ Caucasóide } & \multicolumn{2}{|c|}{ Mulatos } & \multicolumn{2}{|c|}{ Negros } & \multirow[b]{2}{*}{$n \cdot$} & \multirow[b]{2}{*}{$\%$} & \multirow[b]{2}{*}{ p* } \\
\hline & $\mathrm{n} \cdot$ & $\%$ & $n \cdot$ & $\%$ & n & $\%$ & & & \\
\hline $\begin{array}{c}\mathrm{ABO} \\
0 \\
\mathrm{~A} \\
\mathrm{~B} \\
\mathrm{AB}\end{array}$ & $\begin{array}{l}388 \\
329 \\
96 \\
21\end{array}$ & $\begin{array}{c}(46,52) \\
(39,45) \\
(11,51) \\
(2,52)\end{array}$ & $\begin{array}{c}440 \\
245 \\
114 \\
28\end{array}$ & $\begin{array}{c}(53,20) \\
(29,63) \\
(13,78) \\
(3,39)\end{array}$ & $\begin{array}{c}384 \\
256 \\
133 \\
28\end{array}$ & $\begin{array}{c}(47,94) \\
(31,96) \\
(16,60) \\
(3,50)\end{array}$ & $\begin{array}{c}1,212 \\
830 \\
343 \\
77\end{array}$ & $\begin{array}{c}(49,23) \\
(33,71) \\
(13,93) \\
(3,13)\end{array}$ & $\begin{array}{c}p=0,016 \\
p<0,001 \\
p=0,012 \\
p>0,05\end{array}$ \\
\hline $\begin{array}{ll}\mathrm{P} & \\
& \mathrm{P} 1+ \\
& \mathrm{P} 1-\end{array}$ & $\begin{array}{l}665 \\
169\end{array}$ & $\begin{array}{l}(79,74) \\
(20,26)\end{array}$ & $\begin{array}{l}705 \\
122\end{array}$ & $\begin{array}{l}(85,25) \\
(14,75)\end{array}$ & $\begin{array}{c}726 \\
75\end{array}$ & $\begin{array}{c}(90,64) \\
(9,36)\end{array}$ & $\begin{array}{c}2,096 \\
366\end{array}$ & $\begin{array}{l}(85,13) \\
(14,87)\end{array}$ & $p<0,001$ \\
\hline $\begin{array}{l}\text { Lutheran } \\
\qquad \begin{array}{l}\text { Lu(a+b-) } \\
\text { Lu(a+b+) } \\
\text { Lu(a- b+) } \\
\text { Lu(a- b-) }\end{array}\end{array}$ & $\begin{array}{c}2 \\
58 \\
774 \\
0\end{array}$ & $\begin{array}{c}(0,24) \\
(6,95) \\
(92,81) \\
(0,00)\end{array}$ & $\begin{array}{c}1 \\
53 \\
773 \\
0\end{array}$ & $\begin{array}{c}(0,12) \\
(6,41) \\
(93,47) \\
(0,00)\end{array}$ & $\begin{array}{c}1 \\
50 \\
750 \\
0\end{array}$ & $\begin{array}{c}(0,12) \\
(6,24) \\
(93,63) \\
(0,00)\end{array}$ & $\begin{array}{r}4 \\
161 \\
2,297 \\
0\end{array}$ & $\begin{array}{c}(0,16) \\
(6,54) \\
(93,30) \\
(0,00)\end{array}$ & $\begin{array}{l}p>0,05 \\
p>0,05 \\
p>0,05 \\
p>0,05\end{array}$ \\
\hline $\begin{array}{l}\text { Kell } \\
\qquad \begin{array}{l}\text { K+k- } \\
K+k+ \\
K-k+ \\
\text { Kp(a+b-) } \\
\text { Kp(a+b+) } \\
\text { Kp(a- b+) } \\
\text { Js(a+b-) } \\
\text { Js(a+b+) } \\
\text { Js(a-b+) }\end{array}\end{array}$ & $\begin{array}{c}5 \\
61 \\
768 \\
4 \\
18 \\
812 \\
1 \\
3 \\
714\end{array}$ & $\begin{array}{c}(0,60) \\
(7,31) \\
(92,09) \\
(0,48) \\
(2,16) \\
(97,36) \\
(0,14) \\
(0,42) \\
(99,44)\end{array}$ & $\begin{array}{c}2 \\
33 \\
792 \\
3 \\
3 \\
821 \\
4 \\
18 \\
592\end{array}$ & $\begin{array}{c}(0,24) \\
(3,99) \\
(95,77) \\
(0,36) \\
(0,36) \\
(99,27) \\
(0,65) \\
(2,93) \\
(96,42)\end{array}$ & $\begin{array}{c}6 \\
17 \\
778 \\
1 \\
3 \\
797 \\
0 \\
50 \\
599\end{array}$ & $\begin{array}{c}(0,75) \\
(1,21) \\
(97,13) \\
(0,12) \\
(0,37) \\
(99,50) \\
(0,00) \\
(7,70) \\
(92,30)\end{array}$ & $\begin{array}{c}13 \\
111 \\
2,338 \\
8 \\
24 \\
2,430 \\
5 \\
71 \\
1,905\end{array}$ & $\begin{array}{c}(0,53) \\
(1,21) \\
(97,13) \\
(0,33) \\
(0,97) \\
(98,70) \\
(0,26) \\
(3,58) \\
(96,16)\end{array}$ & $\begin{array}{l}p>0,05 \\
p<0,001 \\
p<0,001 \\
p>0,05 \\
p<0,001 \\
p<0,001 \\
p>0,05 \\
p<0,001 \\
p<0,001\end{array}$ \\
\hline $\begin{array}{l}\text { Lew is } \\
\qquad \begin{array}{l}\text { Le }(a+b-) \\
\text { Le }(a+b+) \\
\text { Le(a- } b+) \\
\text { Le(a- b-) }\end{array}\end{array}$ & $\begin{array}{c}130 \\
10 \\
629 \\
65\end{array}$ & $\begin{array}{c}(15,50) \\
(1,20 \\
(75,42) \\
(7,79)\end{array}$ & $\begin{array}{c}160 \\
8 \\
504 \\
155\end{array}$ & $\begin{array}{c}(19,35) \\
(0,97) \\
(60,94) \\
(18,74)\end{array}$ & $\begin{array}{c}148 \\
8 \\
449 \\
196\end{array}$ & $\begin{array}{c}(18,48) \\
(1,00) \\
(56,05) \\
(24,47)\end{array}$ & $\begin{array}{c}438 \\
26 \\
1,582 \\
416\end{array}$ & $\begin{array}{c}(17,79) \\
(1,05) \\
(64,26) \\
(16,89)\end{array}$ & $\begin{array}{l}p>0,05 \\
p>0,05 \\
p<0,001 \\
p<0,001\end{array}$ \\
\hline $\begin{array}{l}\text { Duffy } \\
\text { Fy(a+b-) } \\
\text { Fy(a+b+) } \\
\text { Fy(a-b+) } \\
\text { Fy(a-b-) }\end{array}$ & $\begin{array}{c}165 \\
345 \\
315 \\
9\end{array}$ & $\begin{array}{c}(19,78) \\
(41,37) \\
(37,77) \\
(1,08)\end{array}$ & $\begin{array}{l}139 \\
182 \\
238 \\
268\end{array}$ & $\begin{array}{l}(16,81) \\
(22,00) \\
(28,78) \\
(32,41)\end{array}$ & $\begin{array}{c}112 \\
13 \\
140 \\
536\end{array}$ & $\begin{array}{c}(13,98) \\
(1,62) \\
(17,48) \\
(66,92)\end{array}$ & $\begin{array}{l}416 \\
540 \\
693 \\
536\end{array}$ & $\begin{array}{l}(16,90) \\
(21,93) \\
(28,15) \\
(33,02)\end{array}$ & $\begin{array}{l}p>0,05 \\
p<0,001 \\
p<0,001 \\
p<0,001\end{array}$ \\
\hline $\begin{array}{l}\text { Kidd } \\
\qquad \begin{array}{l}\text { Jk(a+b-) } \\
\text { Jk(a+b+) } \\
\text { Jk(a-b+) } \\
\text { Jk(a-b-) }\end{array}\end{array}$ & $\begin{array}{c}222 \\
412 \\
195 \\
5\end{array}$ & $\begin{array}{c}(26,62) \\
(49,40) \\
(23,38) \\
(0,50)\end{array}$ & $\begin{array}{c}373 \\
338 \\
115 \\
2\end{array}$ & $\begin{array}{c}(44,99) \\
(40,87) \\
(13,90) \\
(0,24)\end{array}$ & $\begin{array}{c}398 \\
312 \\
90 \\
1\end{array}$ & $\begin{array}{c}(49,69) \\
(38,95) \\
(11,24) \\
(0,12)\end{array}$ & $\begin{array}{c}992 \\
1,062 \\
400 \\
8\end{array}$ & $\begin{array}{c}(49,69) \\
(43,13) \\
(11,24) \\
(0,33)\end{array}$ & $\begin{array}{l}p<0,001 \\
p<0,001 \\
p<0,001 \\
p>0,05\end{array}$ \\
\hline
\end{tabular}

- $n=$ número de indivíduos estudados $\mathrm{p}=$ estimado pelo teste de Qui-quadrado de Pearson

No sistema Lewis, o fenótipo Le(a- b-) foi mais encontrado em negróides (negros=24,47\%; mulatos $=18,74 \%)$ do que em caucasóides $(7,79 \%)$, $\mathrm{p}<0,0001$, como já havia sido observado anteriormente $(26,27,28)$. 0 encontro do raro fenótipo Le $(a+b+)$ nas três populações por nós estudadas (caucasóides $=1,20 \%$; mulatos $=0,97 \%$; negros $=1,00 \%$ ), pode, em parte, ser explicado pelo fato de termos utilizado soros anti-Le e anti$\mathrm{Le}^{\mathrm{b}}$ de origem monoclonal altamente potentes (29). A freqüência do fenótipo Le $(a+b+)$ é mais comum em outras populações, tais como japoneses, 
tailandeses, polinésios, sendo reportado uma freqüência de até $25 \%$ em chineses de Taiwan (28).

Em relação à freqüência fenotípica do antígeno $\mathrm{P}_{1}$, os dados por nós observados na população caucasóide $(79,74 \%)$ são muito próximos do encontrado em caucasóides norte- americanos (77,05\%) (30) e escoceses (79,15\%) (31). Em negros, obtivemos em nosso estudo $(90,64 \%)$ resultados semelhantes aos encontrados em negros norte-americanos (93,10\%) (31).

O percentual de indivíduos caucasóides com fenótipo ddccee (10.97\%) está próximo

Tabela 2. Frequências fen otípicas do sistema de grupo sangüíneo MNS em doadores de sangue Caucasóides, Mulatos e Negros

\begin{tabular}{|c|c|c|c|c|c|c|c|c|c|}
\hline & \multicolumn{6}{|c|}{ População estudada } & \multicolumn{3}{|c|}{ TOTAL } \\
\hline \multirow{2}{*}{$\begin{array}{l}\text { Sistema de grupo } \\
\text { sangüíneo MNS }\end{array}$} & \multicolumn{2}{|c|}{ Caucasóide } & \multicolumn{2}{|c|}{ Mulatos } & \multicolumn{2}{|c|}{ Negros } & \multirow[b]{2}{*}{$n \cdot$} & \multirow[b]{2}{*}{$\%$} & \multirow[b]{2}{*}{ p* } \\
\hline & $n$ & $\%$ & $n$ & $\%$ & $n$ & $\%$ & & & \\
\hline$M+N-S+S-$ & 49 & $(5,88)$ & 27 & $(3,26)$ & 26 & $(3,25)$ & 102 & $(4,14)$ & $p<0,001$ \\
\hline$M+N-S-S+$ & 89 & $(10,67)$ & 107 & $(12,94)$ & 126 & $(15,73)$ & 322 & $(15,73)$ & $p<0,001$ \\
\hline $\mathrm{M}+\mathrm{N}-\mathrm{S}+\mathrm{S}+$ & 104 & $(12,47)$ & 99 & $(11,97)$ & 93 & $(11,61)$ & 296 & $(12,02)$ & $p>0,05$ \\
\hline $\mathrm{M}+\mathrm{N}+\mathrm{S}+\mathrm{S}-$ & 36 & $(4,32)$ & 36 & $(4,35)$ & 32 & $(4,00)$ & 104 & $(4,22)$ & $p>0,05$ \\
\hline $\mathrm{M}+\mathrm{N}+\mathrm{S}-\mathrm{S}+$ & 209 & $(25,06)$ & 233 & $(28,17)$ & 237 & $(29,59)$ & 679 & $(27,58)$ & $p>0,05$ \\
\hline $\mathrm{M}+\mathrm{N}+\mathrm{S}+\mathrm{S}+$ & 174 & $(20,86)$ & 156 & $(18,86)$ & 129 & $(16,10)$ & 459 & $(18,65)$ & $p<0,005$ \\
\hline$M-N+S+s-$ & 10 & $(1,20)$ & 9 & $(1,09)$ & 9 & $(1,12)$ & 28 & $(1,14)$ & $p>0,05$ \\
\hline$M-N+S-S+$ & 112 & $(13,43)$ & 113 & $(13,66)$ & 111 & $(13,86)$ & 336 & $(13,65)$ & $p>0,05$ \\
\hline $\mathrm{M}-\mathrm{N}+\mathrm{S}+\mathrm{S}+$ & 51 & $(6,12)$ & 47 & $(5,68)$ & 30 & $(3,75)$ & 128 & $(5,20)$ & $p>0,05$ \\
\hline$M+N+S-S-U-$ & 0 & $(0,00)$ & 0 & $(0,00)$ & 7 & $(0,87)$ & 7 & $(0,28)$ & $p<0,001$ \\
\hline$M+N+S-s-U+$ & 0 & $(0,00)$ & 0 & $(0,00)$ & 1 & $(0,12)$ & 1 & $(0,04)$ & $p>0,05$ \\
\hline TOTAL & \multicolumn{2}{|c|}{100,0} & \multicolumn{2}{|c|}{100,0} & \multicolumn{2}{|c|}{100,0} & \multicolumn{2}{|c|}{2,462} & $p>0,05$ \\
\hline
\end{tabular}

- $n=$ número de indivíduos estudados $\mathrm{p}=$ estimado pelo teste de Qui-quadrado de Pearson

Tabela 3. Freqüências fenotípicas do sistema de grupo sangüíneo Rh em doadores de sangue Caucasóides, Mulatos e Negros

\begin{tabular}{|c|c|c|c|c|c|c|c|c|c|}
\hline \multirow{3}{*}{ Fenótipo } & \multicolumn{9}{|c|}{ População estudada } \\
\hline & \multicolumn{2}{|c|}{ Caucasóide } & \multicolumn{2}{|c|}{ Mulatos } & \multicolumn{2}{|c|}{ Negros } & \multirow[t]{2}{*}{$n$} & \multirow[t]{2}{*}{$\%$} & \multirow[t]{2}{*}{$p^{*}$} \\
\hline & & $\mathrm{n}$ & $\% \mathrm{n}$ & $\%$ & $\mathrm{n}$ & $\%$ & & & \\
\hline ddccee & 85 & $(10,19)$ & 51 & $(6,17)$ & 37 & $(4,62)$ & 173 & $(1,50)$ & $p<0,001$ \\
\hline Dccee & 69 & $(8,27)$ & 183 & $(22,13)$ & 293 & $(36,58)$ & 545 & $(22,14)$ & $p<0,001$ \\
\hline ddccEe & 0 & $(0,00)$ & 4 & $(0,48)$ & 1 & $(0,12)$ & 5 & $(0,20)$ & $p>0,05$ \\
\hline DccEe & 116 & $(13,91)$ & 107 & $(12,94)$ & 109 & $(13,61)$ & 332 & $(13,08)$ & $p>0,05$ \\
\hline DccEE & 20 & $(2,40)$ & 21 & $(2,54)$ & 13 & $(1,62)$ & 54 & $(2,19)$ & $p>0,05$ \\
\hline ddCcee & 4 & $(0,48)$ & 9 & $(1,09)$ & 7 & $(0,87)$ & 20 & $(0,81)$ & $p>0,05$ \\
\hline DCcee & 311 & $(37,29)$ & 283 & $(34,22)$ & 234 & $(29,21)$ & 828 & $(33,63)$ & $p<0,01$ \\
\hline ddCcEe & 1 & $(0,12)$ & 0 & $(0,00)$ & 0 & $(0,00)$ & 1 & $(0,04)$ & $p>0,05$ \\
\hline DCcEe & 87 & $(10,43)$ & 90 & $(10,88)$ & 60 & $(7,49)$ & 237 & $(9,63)$ & $p>0,05$ \\
\hline DCCEE & 2 & $(0,24)$ & 1 & $(0,12)$ & 2 & $(0,24)$ & 5 & $(0,20)$ & $p>0,05$ \\
\hline DCCee & 123 & $(14,75)$ & 69 & $(9,34)$ & 36 & $(4,49)$ & 228 & $(9,26)$ & $p<0,001$ \\
\hline DCCEe & 3 & $(0,36)$ & 1 & $(0,12)$ & 3 & $(0,37)$ & 7 & $(0,28)$ & $p>0,05$ \\
\hline$D C C^{\mathrm{w}} \mathrm{EE}$ & 0 & $(0,00)$ & 0 & $(0,00)$ & 1 & $(0,12)$ & 1 & $(0,04)$ & $p>0,05$ \\
\hline $\mathrm{DCC}^{\mathrm{w}} \mathrm{Ee}$ & 0 & $(0,00)$ & 2 & $(0,24)$ & 0 & $(0,00)$ & 2 & $(0,08)$ & $p>0,05$ \\
\hline$D C C^{w}$ ee & 10 & $(1,20)$ & 6 & $(0,73)$ & 5 & $(0,62)$ & 21 & $(0,85)$ & $p>0,05$ \\
\hline$D C^{w} C^{w} E E$ & 2 & $(0,24)$ & 0 & $(0,00)$ & 0 & $(0,00)$ & 2 & $(0,08)$ & $p>0,05$ \\
\hline$D C^{w} C E E$ & 1 & $(0,12)$ & 0 & $(0,00)$ & 0 & $(0,00)$ & 1 & $(0,04)$ & $p>0,05$ \\
\hline
\end{tabular}

- $n=$ número de indivíduos estudados $p=$ estimado pelo teste de Qui-quadrado de Pearson os fenótipos DCCEE, ddCCee, ddccEE não foram encontrados neste estudo 
daqueles já descritos na literatura por (8) em indivíduos brancos na cidade de Belém, Estado do Pará $(9,1 \%)$ entretanto, inferior ao já reportado internacionalmente 15\% (24). Para mulatos, a freqüência relativa do fenótipo Rh-negativo $(7,82 \%)$ foi menor que 0 observado por Salaru e Otto (2), na cidade de São Paulo, (9,09\%). Em negros, nossos dados para o fenótipo ddccee $(5,66 \%)$ foram superiores ao já reportados anteriormente em São Paulo (33) e na Amazônia (34). A diferença encontrada em negros pode ser explanada pela possibilidade de influência genética indígena que possa ter o ocorrido nos estudos conduzidos pelos dois autores supra referidos, uma vez que os índios são em sua quase totalidade Rh positivos (20). Quando comparamos nossos resultados para o fenótipo Rh negativo em negros com os da literatura internacional, notamos que nossa casuística foi próxima da observada em negros americanos $(6,1 \%)$

0 fenótipo Dccee é considerado um dos marcadores da raça negra (35). Notamos que os nossos dados para o fenótipo Dccee em caucasóides $(8,28 \%)$ foram semelhantes encontrados em outros estudos brasileiros (2, $36)$, porém, mais freqüentes que os descritos em população norte-americana $(2,3 \%)(29,30)$. Em negros brasileiros, os resultados por nós observados para o fenótipo Dccee $(36,85 \%)$ foi superior ao encontrado por Venturelli e de Moraes (35), 24,84\%, porém inferior aos descritos por Salaru e Otto (41,79\%) (2) e em negros norte-americanos $(47,8 \%)(27)$.

Interessante notar que o raro complexo gênico que produz $C^{w}$ e $C$ mas não $C$ levando ao fenótipo $C^{w}{ }^{w} D E E$ (37), foi identificado em um doador caucasóide.

Encontramos em nossa casuística um doador caucasóide inicialmente identificado como Dc- homozigoto, que não apresentava expressão dos antígenos $\mathrm{E}$ ou e pelos testes de rotina. Após extensos estudos realizados, Huang et al.(38) (1997) concluiu que o transcrito Rhce apresentava uma deleção no exon 5 do "triplet" AGA que codifica para Arginina na posição 229 (Arg229). Outros estudos mostraram posteriormente que a deleção da Arg229 estava correlacionada com uma forma de supressão acentuada da expressão do antígeno e (39). Estes resultados foram os primeiros a estabelecer que a deleção de um "triplet" pode causar uma variação genética no polimorfismo de grupos sangüíneos.

São poucos os estudos nacionais e internacionais, nos quais grande número de indivíduos foram testados simultaneamente com o emprego de soros anti-Kell e anti-Cellano (40). A freqüência fenotípica $K+k+$ por nós encontrada para caucasóides $(7,31 \%)$ e para negros $(1,21 \%)$ foi semelhante a mencionada por em norte-americanos (27). Na nossa casuística, cerca de 0,53\% dos doadores apresentaram fenótipo $\mathrm{K}+\mathrm{k}$-; valor este superior ao referido na literatura, que é de $0,2 \%$ em caucasianos e $<0,1 \%$ em negros (38).

Em relação ao estudo dos antígenos $\mathrm{Kp}^{\mathrm{a}}$ e K ${ }^{b}$, não encontramos trabalhos na literatura nacional e são escassos os existentes na literatura internacional (39), sendo pouco conhecida a freqüência destes antígenos em outros grupos raciais que não os caucasóides. Não encontramos nenhum estudo sobre os antígenos $\mathrm{Kp}^{\mathrm{a}}$ e $\mathrm{Kp} \mathrm{p}^{\mathrm{b}}$ em mulatos. $\mathrm{O}$ fenótipo $\mathrm{Kp}(\mathrm{a}+\mathrm{b}-)$ considerado raro, foi encontrado em $0,48 \%$ dos caucasóides na nossa casuística, valor este superior ao observado em caucasóides norte-americanos $(0,001)$ (29). Tivemos a oportunidade de encontrar um doador negro com o fenótipo $\mathrm{Kp}(a+b-)$ , sendo que não havia sido relatado na literatura até então a ausência do antígeno $\mathrm{Kp}^{\mathrm{b}}$ em negros (39). Tal resultado, devido a raridade foi repetido e por nós confirmado através da fenotipagem para os antígenos $\mathrm{Kp}^{\mathrm{a}}$ e Kp com soros de diferentes procedências e através de testes de adsorsão e eluição. Infelizmente, não pudemos estudar os familiares do doador em questão, para melhor compreender este achado.

$\mathrm{Na}$ nossa casuística, apenas $7,70 \%$ dos negros apresentaram fenótipo $\mathrm{Js}(a+b+)$, valor este bastante inferior quando comparado com o descrito na literatura, que é de cerca de $18,4 \%$ (41). Um raro caso do fenótipo Js(a+ b-) foi observado em doador caucasóide, sendo a freqüência reportada para este fenótipo em caucasóides inferior a 0,1\% (39). 
Tal resultado foi repetido e por nós confirmado através da fenotipagem com soros anti-Js ${ }^{a}$ e anti-Js de diferentes procedências e por testes de adsorsão e eluição. 0 encontro deste fenótipo talvez possa ser explicado por uma influência genética negra em ancestrais anteriores aos avós paternos ou maternos. Entretanto, tal doador não apresentava nenhum outro marcador eritrocitário de raça negra como grupo sangüíneo $B, c c D$ ee, Fy(ab-), S-s- (40).

Para o sistema de grupo sangüíneo Kidd, à exceção do fenótipo Jk(a- b-) as freqüências fenotípicas por nós observadas em caucasóides foram similares às descritas em caucasóides norte-americanos por (27) e em portugueses (42). Em negros, nossos resultados para 0 fenótipo Jk(a- $b+)$, foram superiores $(11,24 \%)$ quando comparados com os dados ap resentados em negros norte-americanos (8,1\%) (43). Em nossa casuística encontramos oito casos $(0,33 \%)$ com fenótipo Jk(a-b-) o que é raro em caucasóides e em negros (38), pois é encontrado numa freqüência inferior a $0,01 \%$ nestas populações, muito embora em indivíduos provenientes da Ásia e da Polinésia (44) 0 fenótipo Jk(a- b-) tenha sido encontrado numa ocorrência maior variando de $0,27 \%$ a 1,45\%. 0 fenótipo Jk(a- b-) também foi descrito em índios do Mato Grosso (45), sugerindo talvez que uma influência genética indígena anterior aos avós paternos e maternos dos participantes justifiquem este achado em nosso estudo, pois dos oito casos analisados com este fenótipo, três são procedentes do Estado do Mato Grosso. O fenótipo Jk (a- b-) nos oito casos por nós encontrados apresentaram teste de lise retardada quando expostos à uréia $2 M$, 0 que é característico deste fenótipo (45).

Em caucasianos europeus, o fenótipo Fy (a- b-) não foi encontrado em dois estudos populacionais de grande amostragem (24), mas foi por nós observado em 1,08\% e em caucasóides norte-americanos (1,3\%). 0 fenótipo Fy (a- b-), que é considerado um dos marcadores de raça negra, foi encontrado em $66,92 \%$ dos negros por nós estudados, dado este superior ao compilado em negros norteamericanos $(60,8 \%)(27)$, porém inferior ao descrito para negros africanos (88\%) (46).
Os resultados para o sistema de grupo sangüíneo MNS foram semelhantes aos já descritos na literatura internacional (24). Nós também testamos os doadores S-s- para o antígeno $U$, tendo sido os nossos achados $(0,99 \%$ em negros) dentro dos valores já descritos e compilados por Issitt em negros norte-americanos e em negros africanos (0,27 a 1,4\%). Nenhum caso de $U^{\text {var }}$ (38) foi observado em nosso estudo.

A maioria das fenotipagens do sistema Lutheran relatada em estudos populacionais foram feitas usando somente anti-Lu ${ }^{\mathrm{a}}$. Nossos resultados, empregando soros anti-Lu ${ }^{a}$ e antiLu foram similares aos já descritos na literatura em caucasóides (48). De acordo com Walker (17), não existiam dados suficientes na literatura para o cálculo da freqüência do sistema Lutheran em negros. Na nossa casuística não foram encontradas diferenças estatisticamente significantes entre caucasóides, mulatos e negros para o grupo sangüíneo Lutheran.

A importância do conhecimento das diferenças nas freqüências dos grupos sangüíneos entre as populações estudadas pode ser refletida na agilização do encontro de unidades negativas de sangue raro, consequentemente, 0 trabalho na procura de sangue raro será racionalizado, possibilitando inclusive o cálculo da estimativa de encontro de unidades negativas para os principais antígenos eritrocitários a partir de dados brasileiros.

Esta pesquisa possibilitou um melhor conhecimento da nossa população quanto a freqüência dos grupos sangüíneos, contribuindo para um aprimoramento no exercício da medicina transfusional no nosso país.

\section{Blood groups in caucasian and black blood donors from São Paulo, Brazil}

Marcia C. Z. Novaretti, Pedro E. Dorlhiac-Llacer, Dalton A.F. Chamone

\section{Abstract}

Despite the fact Mulattos (individuals resulting from admixture of Caucasian and Black individuals) represent one of the most common racial mixed individuals not only in Brazil but in many other countries, there is little 
information regarding the distribution of blood groups among them. We studied 2,462 blood donors classified as Caucasian, Mulattos e Blacks according to their anthropological characteristics as well as to their ancestry information. Phenotype frequencies were studied in the ABO, MNS, P, Rh, Lutheran, Kell, Lewis, Duffy e Kidd blood group systems. We did not find significant statistically difference between Blacks and Mulattos for the majority of the blood groups systems here reported, excepting of $P$ positive, Dccee, Le(a-b-), $J s(a+b+), J s(a-b+), F y(a-b-), F y(a+b+)$ e Fy( $a-$ $b+)$. On the other he there was a significant difference between Caucasian and Blacks for the following red blood cells phenotypes: $A, B$, $\mathrm{M}+\mathrm{N}-\mathrm{S}+\mathrm{S}-, \mathrm{M}+\mathrm{N}-\mathrm{S}-\mathrm{S}+, \mathrm{P} 1$ positivo, ddccee, Dccee, Dccee, DCCee, DccEe, $\mathrm{K}+\mathrm{k}+, \mathrm{K}-\mathrm{k}+$, $K p(a-b+), K p(a+b+), J s(a-b+), J s(a+b-)$, Le( $a-$ $b+)$, Le(a-b-), Fy(a-b+), Fy(a+b+), Fy(a-b-), $J k(a+b-), J k(a+b+)$ e Jk( $a-b+)$. Conclusion: As expected, the results for the Mulattos group were intermediate between Caucasian and Blacks, with strong Negroid influence.

Rev.bras.hematol.hemoter., 2000, 22(1):23-32

Key words: blood groups, statistics, blood donors

Agradeço ao Prof. Dr. Paulo Otto pelos sábios conselhos e pelo desenvolvimento de software específico e aos Drs. Marion E. Reid e Graeme Woodfield pela revisão.

\section{Referências bibliográficas}

1. Salzano F.M. Genetic polymorphisms in Brazilian populations. In: Salzano F.M.. (ed.): The ongoing evolution of Latin American Populations. Springfield: Charles C. Thomas, 1971, p 631-655.

2. Salaru N.N., Otto P. Blood groups in a large sample from the city of São Paulo (Brazil): allele and haplotype frequencies for MNSs, Kell-Cellano, Rh and ABO systems. Rev Bras Genet 1989,12: 625-643.

3. IBGE: Instituto Brasileiro de Geografia e Estatística, www.ibge.gov.br, 1998.

4. Conceição M.M., Salzano F.M., Franco M.H.P. et al. Demography genetics and race admixture in Aracaju, Brazil. Rev Bras Genet 1987, 10: 313-331.

5. Montenegro L. Blood group in tucano indians. Hum Biol. 1967, 9: 89-92.

6. Long J.C, Smouse P.E. Tribal gene flow between the Yeçuana and Yanomana: Genetic Analysis of an admixed village. Am J Phys Anthropol, 1983, 61: 411-22.

7. Rosa V.L., Salzano F.M., Franco, M.H.L.P. et al. Blood genetic studies in five Amazonian populations. Rev Bras Genet, 1984, 7: 569-82.

8. Guerreiro J.F., Chautard-Freire-Maia. ABO and Rh blood groups, migration and estimates of racial admixture for the population of Belém, State of Pará, Brazil. Rev Bras Genet, 1988, 11: 171-86.

9. De Lucca E.J. Pesquisas genéticas na população de Humaitá (AM). I Grupos Sanuíneos ABO, Rh e MN. Rev Bras Med 1975, 32:397-400.

10. Lima L.M.A., Callado M.R.M., Santos J.A. Curso de Imunohematologia. Botucatu: Unesp, 1992.

11. Salzano F.M., Weimer T.A., Franco M.H.L.P. et al. Population structureand blood genetics of the Pacaás Novas Indians of Brazil. Ann Hum Biol, 1985, 12: 241-9.

12. Salzano F.M., Black F.L., Callegari-Jacques S.M. Blood genetic systems in four Amazonian Tribes. Am J Phys Anthropol, 1991, 85: 51-60.

13. BRASIL. Ministério da Saúde. Portaria no.1.376, 19 de novembro de 1993. Normas técnicas para Coleta, Processamento e Transfusão de Sangue, Componentes e Hemoderivados. Diário Oficial [República Federativa do Brasil], Brasília, 131, n.229:18405-15, 2 dez. 1993, seção 1.

14. Casey A.E., Hale K., Casey J.G. et al. Blood group-anthropometric profiles of constitutional variation and susceptibility to disease. Ala J Med Sci, 1978, 15: 353-62.

15. Franco R.F., Simões B.P., Zago, M.A. Relative frequencies of the two $O$ alleles of the histoblood group ABH system in different racial groups. Vox Sang 1995, 69: 50-2.

16. Palatnik M., Sá e Benevides M.J.F. de; Salzano F.M. ABH salivary secretion and white/negro gene flow in a Brazilian 
population. Hum Biol, 1969, 41: 84-96.

17. Cliquet, M. G. ( Fundação Pró-Sangue / Hemocentro de São Paulo, 1990)

18. Walker R.H. (ed.) Technical Manual, 11 ed. Bethesda, Maryland: American Association of Blood Banks, 1993.

19. Fleiss J.L. Statistical methods for rates and proportions. 2 ed. New York: John Wiley \& sons, 1981, p138-58.

20. Black F.L., Santos S.E.B., Salzano F.M., Mestriner M.A., Pandey J.P. Genetic variation within the Tupi linguistic group: new data on three Amazonian tribes. Ann Hum Biol 1988, 15: 337-51.

21. Matson G.A., Sutton E., Pessoa E.M. et al. Distribution of hereditary blood groups among indians in South America. Am J Phys Anthropol 1970, 28: 303-30.

22. Salzano F.M. Mohrenweiser H., Gershowitz $\mathrm{H}$., et al. New studies on the Macushi Indians of Northern Brazil. Am Hum Biol 1984, 11: 337-50.

23. Saldanha A.S. ABO blood groupsand salivary secretion of $A B H$ substances among three racial groups in São Paulo City. Rev Bras Genet 1982, 5: 175-86.

25. Race R.R., Sanger R. Blood groups in Man. 6 ed. Blackwell Scientific Publications, Oxford, 1975.

26. Moltham L. Lewis Phenotype of American Caucasian, American Negroes and their children. Vox Sang 1980, 39: 327-330.

27. Palatnik M., Laranjeira, N.S.M., Simões, M.L.M.S et al. Distribution of the Lewis and Secretor Trait in Rio de Janeiro, Brazil. Rev Bras Genet 1988, 11: 187-92.

28. Gaensleen R.E., Bell S.C., Lee H.C. Distributions of gen etic markers in the United Sates populations: I blood group and secretor systems. J Forensic Sci 1987, 32: 1016-57.

29. Lin M, Broadberry R.E. Lewis phenotypes in Orientals. Vox Sang 1995, 68: 68-9.

30. Shreffler D.C., Sing C.F., Neel J.V. et al. Studies on genetic selection in a completely ascertained caucasian population. I. Frequencies, Age and Sex effects, and phenotype associations for 12 Blood group systems. Am J Hum Genet 1971, 23: 50-63.

31. Clegg E.J., Tills D., Warlow A., Wilkinson J. et al. Blood group variation in the isle of
Lewis. Ann Hum Biol 1985, 12: 345-61.

32. Reed T.E.. Distributions and tests of independence of seven blood group systems in a largemultiracial sample from California.

Am J Hum Genet 1968, 20: 142-50.

33. Seixas A.S.S., Werneck P.S., Plepis Jr M. et al. Incidência e distribuição racial dos grupossanguín eos (sistema ABO) efator Rhna cidade de São Carlos (Estado de São Paulo). "O Hospital", 1968, 74: 277-81.

34. Schneider H., Guerreiro JF, Santos SEB et al. Isolate breakdown in Amazonia- The blacks of the Trombetas River. Rev Bras Genet 1987, 10: 566-74.

35. Sanchez-Mazas A.S., Langsganey A. Common genetic pools between human populations. Hum Genet 1988, 78:161-8.

36. Venturelli L.E., De Morais M.M.J.B. Frequencias gênicas dos sistemas $A B O$, MNSs e Rh em caucasóides e negróides da cidade de Campinas, SP. Rev Bras Genet 1986, 9: 179-85.

37. Daniels G.F. Human Blood groups. Blackwell Science.1995, London. 719 p.

38. Huang, C.H., Reid, M.E., Chen Y., Novaretti M.C.Z. Deletion of ARG 229 in RhCE polipeptide alters expression of RhE and CEassociated Rh6 antigens. Blood 1997 suppl1, 90 (10): 1193.

39. Issitt P.D., Anstee D.J. Applied Blood Group Serology. 4 ed. 1999, Montgomery Scientific Publications, Durham, North Carolina, E.U.A.1208 p.

40. Daniels $G$. The Kell blood group system: Genetics. In: Laird-Fryer B; Daniels G; Levitt J( eds). Blood group systems: Kell,. Arlington, VA: American Association of Blood Banks, 1990, p-1-36.

41. Issitt P.D. Race-related red cell alloantibody problems. Br J BioMed Sci 1994, 51:158-67.

42. Pinheiro M.F., da Costa V.P., de Sousa M.V. et al. Genetic polymorphisms- North of Portugal. Acta Med Leg Soc 1989, 9: 115-27.

43. Mougey T.R. The Kidd blood group system: serology and genetics. In: Pierce, S.R., MacPherson C.R.( eds) Blood Group systems: Duffy, Kidd and Lutheran. Arlington, VA: American Association of blood Banks 1988, 53-71

44. Woodfield D.G., Douglas R., Smith J. et al. 
The Jk(a-b-) phenotype in New Zealand Polynesians. Transfusion 1982, 22: 276-8.

45. Silver R.T., Haber J.M., Kellner A. Evidence for a new allele in the Kidd blood group system in Indians of Northern Mato Grosso, Brazil. Nature 1960, 186 :481.

46. Heaton D.C., McLoughlin K. Jk(a-b-) red blood cells resist urea lysis. Transfusion 1982, 22: 70-1.

47. Beatttie K.M. The Duffy Blood group system: distribution, serology and gen etics.In: Pierce, S.R., MacPherson, C.R., (eds) Blood group systems: Duffy, Kidd and Lutheran. Arlington, VA: American Association of blood Banks, 1988, 1-25.

48. Crawford M.N. The Lutheran Blood group system: serology and genetics. In: Pierce S.R.; MacPherson C.R.(eds) Blood group systems: Duffy, Kidd and Lutheran. Arlington, VA. American Association of Blood Banks, 1988, 93-117.

Recebido: 29/02/2000

Aceito: 10/03/2000 